\title{
Consistent Structural Linearization in
}

\section{Flexible Aircraft Dynamics with Large}

\section{Rigid-Body Motion}

\author{
Henrik Hesse* , Rafael Palacios ${ }^{\dagger}$ \\ Imperial College, London SW7 2AZ, United Kingdom \\ Joseba Murua ${ }^{\ddagger}$ \\ University of Surrey, Guildford GU2 7XH, United Kingdom
}

This paper investigates the linearization, using perturbation methods, of the structural deformations in the nonlinear flight dynamic response of aircraft with slender, flexible wings. The starting point is the coupling of a displacement-based geometrically nonlinear flexible-body dynamics formulation with a 3D unsteady vortex lattice method. This is followed by a linearization of the structural degrees of freedom, which are assumed to be small in a body-fixed reference frame. The translations and rotations of that reference frame and their time derivatives, which describe the vehicle flight dynamics, can still be arbitrarily large. The resulting system preserves all couplings between rigid and elastic motions and can be projected onto a few vibration modes of the unconstrained aircraft with geometrically

${ }^{*}$ Graduate Student, Department of Aeronautics, AIAA Student Member.

${ }^{\dagger}$ Senior Lecturer, Department of Aeronautics (Contact author: rpalacio@imperial.ac.uk), AIAA Member.

${ }^{\ddagger}$ Lecturer, Department of Mechanical Engineering Sciences, AIAA Member. 
nonlinear static deflections at a trim condition. Equally, the unsteady aerodynamics can be approximated on a fixed lattice defined by the deformed static geometry. Numerical studies on a representative high-altitude, longendurance aircraft are presented to illustrate the approach. Results show an improvement compared to those obtained using the mean-axes approximation.

\section{Nomenclature}

Symbols

$C \quad$ coordinate transformation matrix from frame $A$ to $B$

$\mathcal{C} \quad$ global tangent damping matrix

I unit matrix

$\mathcal{K} \quad$ global tangent stiffness matrix

$\mathcal{M} \quad$ global tangent mass matrix

$p \quad$ generalized displacements in modal basis

$Q \quad$ global vector of generalized forces, $\mathrm{N}$

$r \quad$ inertial position of the origin of the body-fixed frame, $\mathrm{m}$

$R \quad$ beam local position vector, $\mathrm{m}$

$s \quad$ length of the arc along beam reference line, $\mathrm{m}$

$t \quad$ physical time, $\mathrm{s}$

T tangential operator

$v \quad$ inertial translational velocity of the body-fixed frame, $\mathrm{m} / \mathrm{s}$

$V \quad$ inertial translational velocity at a beam cross section, $\mathrm{m} / \mathrm{s}$

$x \quad$ state vector

$\beta \quad$ vector of global translational and rotational velocities

$\gamma \quad$ beam local force strain 


\section{Subscripts}

Superscripts

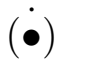

$(\bullet)^{\prime}$ circulation strength of a vortex ring, $\mathrm{m}^{2} / \mathrm{s}$

quaternion for global orientation of the body-fixed frame

vector of nodal displacements and rotations

Euler angles, rad

beam local moment strain, $\mathrm{m}^{-1}$

global displacements and rotations as time integral of $\beta$

beam cross-sectional coordinates, $\mathrm{m}$

matrix of mode shapes

coordinates of the aerodynamic lattice, $\mathrm{m}$

beam local Cartesian Rotation Vector

inertial angular velocity of the body-fixed frame, rad/s

inertial angular velocity at a beam cross section, $\mathrm{rad} / \mathrm{s}$

body-fixed reference frame

local reference frame on flexible members

fluid degrees of freedom

inertial reference frame

rigid-body degrees of freedom

structural degrees of freedom

derivatives with respect to time, $t$

derivatives with respect to length, $s$

skew-symmetric operator

small perturbations around an equilibrium 


\section{Introduction}

The flight dynamics of flexible aircraft have been traditionally studied using linear methods. The standard approach in the flight simulation community is the mean-axes approximation, ${ }^{1-4}$ which decouples the structural and rigid-body dynamics degrees of freedom (DoF) by assuming a superposition of the "free-free"modal vibrations of the structure to the dynamics of a floating frame linked to the aircraft center of gravity. The aerodynamic loads are obtained as the generalized forces corresponding to the mode shapes of the structure, including their rigid-body component. ${ }^{5}$ The resulting equations of motion (EoM), however, neglect some gyroscopic coupling terms (which may have an important effect on both the flight dynamics and the maneuver loads) and are therefore only valid for perturbations about steady flight of relatively stiff vehicles. A more general solution has been proposed using quasi-coordinates,${ }^{6-8}$ which directly solve the fully-coupled EoM of a flexible body (in a body-attached reference frame) under the assumption of small structural deformations.

The coupled aeroelasticity/flight dynamics problem is often linked to large structural deformations and most of the recent work ${ }^{9-14}$ has been concerned with developing fully coupled models to include the effect of geometrically nonlinear elastic deformations on the flight dynamics of flexible aircraft. Typically, the displacements and rotations along a beamtype structure are the primary variables in the numerical solution, where the elastic DoF are defined in terms of a global body-fixed reference frame attached to the reference configuration to include the rigid-body motion of the vehicle. Alternative solutions using directly the beam stress resultants as primary variables ${ }^{13-16}$ or mixed methods based on local velocities and strains (the so-called intrinsic description) $)^{17-19}$ have often provided numerical advantages for aircraft-type geometries.

Whereas geometrically nonlinear static aeroelastic effects can have a significant impact

on the vehicle response, ${ }^{20}$ using full nonlinear models to compute the flight dynamics of very flexible aircraft comes at a numerical burden which makes them less suitable for efficient control design and optimization studies. Model reduction of the system could solve this 
problem but its application is limited here because of the tightly-coupled, nonlinear nature of the flexible-body dynamics subsystem. Recently, Da Ronch et al. ${ }^{21}$ have demonstrated that third-order Taylor expansions with only a few DoF can be obtained directly from veryhigh-dimensional nonlinear aeroelastic models of very flexible wings, but the validity of those approximations can be hard to guarantee far from the reference conditions. In a parallel effort, $\mathrm{Su}$ and $\mathrm{Cesnik}^{22}$ applied a modal approach to the strain-based, geometrically nonlinear beam equations to solve the dynamics of flexible aircraft. However, the reduced system matrices still depended on the modal amplitudes and the computational advantage of that approach was small.

To compute the dynamic response of structures around a geometrically nonlinear static equilibrium, previous work by the first two authors ${ }^{23}$ demonstrated the consistent linearization of the structural DoF in nonlinear flexible-body dynamics problems. This provides a formulation which allows projection of the nonlinear EoM onto the vibration modes of the unconstrained deformed structure at forward flight with possibly large trim deformations. While keeping the nonlinearity in the rigid-body dynamics equations and preserving all couplings between rigid-body and structural dynamics, this approach results in closed-form expressions for all the modal coefficients which only need to be computed once.

The objective of this paper is to exercise this consistent structural linearization on the dynamic analysis of flexible aircraft. Here, the emphasis is on small elastic deformations around the aircraft trim configuration with possibly large static wing deformations. The flexible-body dynamics formulation is coupled with a 3D unsteady vortex lattice method $(\mathrm{UVLM})^{24}$ in the framework for Simulation of High-Aspect-Ratio Planes (SHARP). ${ }^{12,20} \mathrm{Nu}-$ merical examples exercise the proposed formulation on the open-loop response of a representative high-altitude, long-endurance (HALE) aircraft subject to commanded control surface inputs. Comparison to the mean-axes approximation will give an insight into the effect of neglecting the coupling between the elastic deformations and aircraft flight dynamics. 


\section{Flexible-Body Dynamics}

The flexible vehicle will be modeled using geometrically nonlinear composite beam elements on a moving (body-attached) frame of reference. ${ }^{25,26}$ The elastic DoF are the displacements and the Cartesian Rotation Vector (CRV) at the element nodes, with respect to a moving body-attached frame. The nonlinear EoM are then linearized with respect to the elastic DoF using perturbation methods and projected onto a set of elastic modes of the unrestrained system at some reference condition. This will give a compact form of the EoM with large rigid-body motion. The details can be found in Hesse and Palacios ${ }^{23}$ and this section will only present the main results.

\section{II.A. Geometrically Exact Displacement-Based Formulation}

As shown in Figure 1, the deformation of the structure is described in terms of a moving, body-fixed reference coordinate system, $A$, which moves with respect to an inertial (ground) frame, $G$, by the inertial translational and angular velocities, $v_{A}(t)$ and $\omega_{A}(t)$, of its origin. Subscripts are used to indicate the coordinate system in which each vector magnitude is projected. The orientation of the global frame, $A$, with respect to the inertial frame, $G$, is parameterized using quaternions, $\zeta(t)$. The local orientation along the beam reference line is defined by a local coordinate system, $B$, and is parameterized by the CRV, $\Psi(s, t)$. The corresponding coordinate transformation matrix between the global body-fixed reference frame, $A$, and the local deformed frame, $B$, will be referred to as $C(\Psi)$.

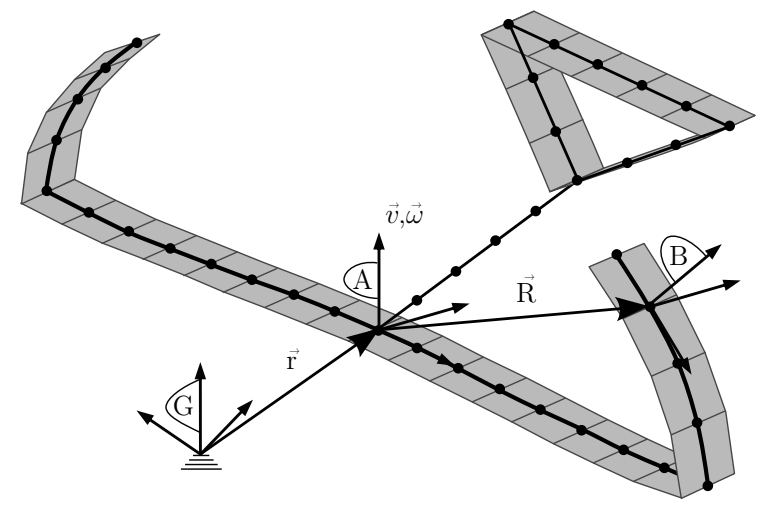

Figure 1: Multi-beam configuration with the definition of reference frames. 
The nodal positions, $R_{A}(s, t)$, and the cross-sectional orientations, $\Psi(s, t)$, thus form the independent set of variables. Note that the elastic displacements are given with respect to the body-fixed frame of reference, $A$, such that the structure is attached to the origin of the $A$ frame (with the corresponding boundary conditions). The rigid-body velocities, $v_{A}$ and $\omega_{A}$, are used to describe the overall motion of the unconstrained vehicle. Hence, the deformation of the reference line going from the undeformed state at $t=0$ to the current state at time $t$ is given by the force and moment strains, ${ }^{27}$ which are defined as

$$
\begin{aligned}
& \gamma(s, t)=C(\Psi(s, t)) R_{A}^{\prime}(s, t)-C(\Psi(s, 0)) R_{A}^{\prime}(s, 0), \\
& \kappa(s, t)=T(\Psi(s, t)) \Psi^{\prime}(s, t)-T(\Psi(s, 0)) \Psi^{\prime}(s, 0),
\end{aligned}
$$

where $(\bullet)^{\prime}$ is the derivative with respect to the length of the arc $s$, and $T(\Psi)$ is the tangential operator. ${ }^{26}$ Analogously, the local translational and angular inertial velocities are obtained, respectively, as

$$
\begin{aligned}
V_{B}(s, t)= & C(\Psi(s, t)) \dot{R}_{A}(s, t)+ \\
& C(\Psi(s, t))\left[v_{A}(t)+\widetilde{\omega}_{A}(t) R_{A}(s, t)\right], \\
\Omega_{B}(s, t)= & T(\Psi(s, t)) \dot{\Psi}(s, t)+C(\Psi(s, t)) \omega_{A}(t),
\end{aligned}
$$

where $(\dot{\bullet})$ is the derivative with respect to time and $(\tilde{\bullet})$ the cross-product (or skew-symmetric) operator. $^{25}$ This kinematic description serves to express the kinetic and potential energy of the system in terms of the beam displacement and rotations. ${ }^{16,26}$ A 2-noded finite-element discretization is then introduced on both variables which gives the discrete form of the nonlinear EoM. If $\eta$ is the column vector with all the nodal displacements and rotations and $\beta^{\top}=\left\{\begin{array}{ll}v_{A}^{\top} & \omega_{A}^{\top}\end{array}\right\}$, the dynamic equations are written as $^{23}$

$$
\mathcal{M}(\eta)\left\{\begin{array}{c}
\ddot{\eta} \\
\dot{\beta}
\end{array}\right\}+\left\{\begin{array}{c}
Q_{g y r}^{S} \\
Q_{g y r}^{R}
\end{array}\right\}+\left\{\begin{array}{c}
Q_{s t i f}^{S} \\
0
\end{array}\right\}=\left\{\begin{array}{c}
Q_{e x t}^{S} \\
Q_{e x t}^{R}
\end{array}\right\}
$$

where structural and rigid-body components (denoted by superscripts $S$ and $R$ ) have been 
identified in the gyroscopic, elastic and external forces. These differential equations couple the nonlinear beam dynamics and the nonlinear rigid-body dynamics through the fully-populated mass matrix, $\mathcal{M}(\eta)$, and the discrete gyroscopic forces, $Q_{g y r}^{S}(\eta, \dot{\eta}, \beta)$ and $Q_{g y r}^{R}(\eta, \dot{\eta}, \beta)$. Since this formulation includes rotational DoF, the mass matrix depends on the current geometry, $\eta$, to account for a change in rotational inertia on the structural and rigid-body dynamics. ${ }^{26}$ In addition to the inertial coupling, additional coupling can occur through follower forces such as aerodynamic loads or thrust. Equation (3) is solved together with the propagation equations that determine the position and orientation of the body-fixed reference frame. ${ }^{28}$ The equations are time-marched using an implicit, constant-acceleration Newmark integration scheme which was modified as in Eq. (7.3.23) in Géradin and Rixen ${ }^{29}$ to introduce controlled positive algorithmic damping.

\section{II.B. Linearization of Elastic Degrees of Freedom in Nonlinear Flexible-Body Dynamics}

The particular case of small structural deformations about a static equilibrium condition is considered next. The possibly large elastic deformations at static equilibrium will be referred to as $\eta$ as before, whereas $\bar{\eta}(t)$ will be the small elastic deformations that occur together with the (not-necessarily small) rigid-body velocities $\beta(t)$. With those assumptions, perturbation of the elastic DoF in Eq. (3) gives linear inertial and elastic terms, which only depend on the deformed shape, whereas the gyroscopic forces require particular attention because of the coupling between rigid-body and elastic states. This results in $^{23}$

$$
\mathcal{M}(\eta)\left\{\begin{array}{c}
\ddot{\bar{\eta}} \\
\dot{\beta}
\end{array}\right\}+\overline{\mathcal{C}}(\eta, \beta)\left\{\begin{array}{l}
\dot{\bar{\eta}} \\
\beta
\end{array}\right\}+\overline{\mathcal{K}}(\eta, \beta)\left\{\begin{array}{l}
\bar{\eta} \\
0
\end{array}\right\}=\bar{Q}_{\text {ext }}(\bar{\eta}, \dot{\bar{\eta}}, \beta, \zeta)
$$

where $\overline{\mathcal{C}}$ and $\overline{\mathcal{K}}$ are the damping and stiffness matrices which originate from the perturbation of $Q_{g y r}$ and $Q_{\text {stif }}$ in the elastic DoF. It is important to note that the mass matrix $\mathcal{M}$ only depends on the static deformations, $\eta$, but the damping and stiffness matrices are also 
functions of the instantaneous rigid-body velocity, $\beta$.

To compute the vibration modes of the unconstrained structure around a geometrically nonlinear deformed reference condition, we can further linearize the dynamic system, Eq. (4), with respect to the rigid-body velocities around the equilibrium $\beta=0$. Note however that the linearization is invariant with respect to translational velocities and the equations determine the dynamics for a trimmed vehicle in forward flight. The homogeneous form of the resulting linear system of coupled EoM will also be the basis for the aeroelastic stability analysis presented in Section III.B.

\section{II.C. Modal Reduction of the Nonlinear System Equations}

It is now possible to write the system of perturbation equations, Eq. (4), in terms of global shape functions by projecting the dynamics equations on the natural modes of the unconstrained structure at a reference condition. These vibration modes are obtained in the body-fixed frame $A$ from the unforced fully-linearized version of Eq. (4), such that

$$
\left\{\begin{array}{c}
\bar{\eta} \\
\bar{\nu}
\end{array}\right\}=\Phi\left\{\begin{array}{c}
p \\
\bar{\nu}^{*}
\end{array}\right\}=\left[\begin{array}{ll}
\Phi_{S S} & \Phi_{S R} \\
\Phi_{R S} & \Phi_{R R}
\end{array}\right]\left\{\begin{array}{c}
p \\
\bar{\nu}^{*}
\end{array}\right\}
$$

where we have introduced the new variable $\bar{\nu}$ with $\dot{\bar{\nu}}=\bar{\beta}$, such that the vector of modal rigid-body displacements and rotations, $\bar{\nu}^{*}$, describes the motion of the body-fixed frame, $A$, and the displacements of this frame due to the elastic mode shapes $\left(\Phi_{R S} \neq 0\right)$. The vector of the projected modal coordinates is $p$ and $\Phi$ is the matrix of the corresponding mode shapes, which include the six zero-frequency rigid-body modes $\left[\begin{array}{ll}\Phi_{S R}^{\top} & \Phi_{R R}^{\top}\end{array}\right]^{\top}$.

Without loss of generality, the origin of the body frame $A$ can be defined to initially coincide with the center of mass $(\mathrm{CM})$ and its axes to be aligned to the principal axes of the structure. This simplifies the rigid-body modes, since $\Phi_{S R}$ and $\Phi_{R R}$ are then a null and a unit matrix, respectively. Note that this is the same initial condition as for a meanaxes description, but $A$ is still a body-fixed frame. The remaining elastic mode shapes, 
$\left[\begin{array}{ll}\Phi_{S S}^{\top} & \Phi_{R S}^{\top}\end{array}\right]^{\top}$, characterize the vibration modes of the unconstrained structure such that $\Phi_{S S}$ are the deformations with respect to frame $A$ and $\Phi_{R S} \neq 0$ accounts for the corresponding rigid-body motion of the body-fixed frame $A$.

However, using this modal basis for the projection of the linear-flexible/nonlinear-rigid EoM, Eq. (4), requires a linear transformation at each sub-iteration to obtain the instantaneous rigid-body velocities needed in the perturbation damping and stiffness matrices. A more convenient basis for projection is

$$
\Phi^{*}=\left[\begin{array}{ll}
\Phi_{S S} & \Phi_{S R} \\
\Phi_{R S} & \Phi_{R R}
\end{array}\right]\left[\begin{array}{cc}
I_{m} & 0 \\
-\Phi_{R S} & I_{6}
\end{array}\right]
$$

where $m$ is the number of elastic modes used for the projection, which relieves the inertial coupling between the rigid-body motion of the vehicle and the elastic deformations. This approach is very similar to that followed by Rodden and Love ${ }^{5}$ to obtain the rigid-body accelerations on the structure in a mean-axes framework and will allow us to separate elastic from rigid-body modes in the following projection of the perturbation flexible-body EoM. Note that in this formulation, the modal basis is computed for the deformed configuration at trimmed flight, which is the same reference condition used for linearization of the elastic DoF.

\section{II.C.1. Modal Reduction of the Perturbation Equations of Motion}

The structural and rigid-body velocities/accelerations can first be expressed in terms of the modal basis, $\Phi^{*}$, such that

$$
\left\{\begin{array}{c}
\dot{\bar{\eta}} \\
\beta
\end{array}\right\}=\Phi^{*}\left\{\begin{array}{c}
\dot{p} \\
\beta
\end{array}\right\} \text { and }\left\{\begin{array}{c}
\ddot{\bar{\eta}} \\
\dot{\beta}
\end{array}\right\}=\Phi^{*}\left\{\begin{array}{c}
\ddot{p} \\
\dot{\beta}
\end{array}\right\}
$$


which is substituted in Eq. (4) to obtain the modal form of the coupled EoM for this system, as

$$
\begin{aligned}
& \Phi^{* \top} \mathcal{M}(\eta) \Phi^{*}\left\{\begin{array}{c}
\ddot{p} \\
\dot{\beta}
\end{array}\right\}+\Phi^{* \top} \overline{\mathcal{C}}(\eta, \beta) \Phi^{*}\left\{\begin{array}{l}
\dot{p} \\
\beta
\end{array}\right\}+ \\
& \Phi^{* \top} \overline{\mathcal{K}}(\eta, \beta) \Phi^{*}\left\{\begin{array}{l}
p \\
0
\end{array}\right\}=\Phi^{* \top} \bar{Q}_{e x t}(\bar{\eta}, \dot{\bar{\eta}}, \beta, \zeta) .
\end{aligned}
$$

The resulting modal EoM describes the arbitrarily-large rigid-body motion of the flexible body subject to small elastic deformations which are captured using the modified vibration modes of the unconstrained structure. Because of the linear mapping of the rigid-body velocities, Eq. (6), the orthogonality property of the resulting vibration modes with respect to the system mass and stiffness matrices is lost. However, the system is already coupled due to the contribution of the gyroscopic forces to the modal damping and stiffness matrices.

The modal damping and stiffness matrices remain functions of the rigid-body DoF, $\beta$. However, it is easy to see that they have, respectively, linear and quadratic dependencies with $\beta$, and that it is possible to write them in terms of third- and fourth-order tensors, ${ }^{23}$

$$
\begin{aligned}
\Phi_{i j}^{*} \overline{\mathcal{C}}_{j k} \Phi_{k l}^{*} & =c_{i l r} \beta_{r}(t), \\
\Phi_{i j}^{*} \overline{\mathcal{K}}_{j k} \Phi_{k l}^{*} & =k_{i l}^{\text {stif }}+k_{i l r s}^{\text {gyr }} \beta_{r}(t) \beta_{s}(t),
\end{aligned}
$$

where we sum over repeated indices and have identified the contributions to the modal stiffness matrix from elastic and gyroscopic forces. The tensors $c$ and $k$ are constant in time and their dimensions are $i, l=\{1, \ldots, m\}$ and $r, s=\{1, \ldots, 6\}$ for $m$ number of modes used in the expansion. These tensors are typically very sparse, and this approach generates efficient numerical solutions that keep the nonlinearities in the rigid-body DoF and all couplings with the linear structure at a low computational cost. 


\section{II.C.2. A Mean-Axes Approximation}

In this section we give a brief description on the implementation of the mean-axes approximation which will be exercised in the numerical studies in Section IV. As it will be seen, the system size is similar to the proposed modal formulation, but it neglects the gyroscopic couplings between the elastic and rigid-body dynamics.

The mean-axes system is enforced by projecting the linear EoM onto free-free modes. ${ }^{4,30}$ Here, we obtain the free-free modes in the inertial frame by solving the eigenvalue problem given by the unconstrained, undamped elastic EoM as, $\mathcal{M}^{S S} \ddot{\eta}^{f}+\mathcal{K}_{\text {stif }}^{S} \eta^{f}=0$, where $\eta^{f}$ is the vector of elastic DoF without enforcing a constrained node at the origin of frame $A$. The corresponding stiffness matrix, $\mathcal{K}_{\text {stif }}^{S}$, is obtained through linearization of the elastic forces, $Q_{\text {stif }}^{S}$. The relative displacements of the flexible body can then be described in terms of mode shapes, $\Phi^{f}$, and generalized displacements, $p^{f}$, such that $\eta^{f}=\sum_{i} \Phi_{i}^{f} p_{i}^{f}$. Due to orthogonality of the free-free modes and if we neglect the contribution of elastic deformations to the gyroscopic forces in Eq. (3), it is possible to solve the mean-axes EoM,

$$
\ddot{p}_{i}^{f}+\omega_{i}^{2} p_{i}^{f}=\left(\Phi_{i}^{f}\right)^{\top} Q_{e x t}^{f}(\bar{\eta}, \beta, \zeta)
$$

separately from the nonlinear rigid-body dynamics EoM, extracted from Eq. (3), as

$$
\mathcal{M}^{R R}(\eta) \dot{\beta}+Q_{g y r}^{R}(\eta, \beta)=Q_{e x t}^{R}(\bar{\eta}, \beta, \zeta)
$$

where $\omega_{i}$ is the vibration frequency of the $i^{\text {th }}$ mode and $\mathcal{M}^{R R}$ the mass matrix of the rigidbody at the CM and principal axes. Neglecting some gyroscopic terms in the damping matrix is a necessary assumption to diagonalize all modal system matrices. The implication of this assumption will be investigated in this paper on a full aircraft application. Note that the resulting set of EoM can still be coupled if the external forces, $Q_{\text {ext }}$, such as aerodynamic loads, not only depend on rigid-body velocities, $\beta$, and orientation, $\zeta$, of the aircraft, but also on the instantaneous geometry due to deformation of the lifting surfaces, $\bar{\eta}$. 


\section{Coupled Aeroelasticity and Flight Dynamic Models}

The presented flexible-body dynamics description has been implemented in the framework for Simulation of High Aspect Ratio Planes (SHARP) ${ }^{12,20}$ to study flexible aircraft, including static aeroelastic analyses, trim, linear stability analyses, and fully nonlinear time-marching simulations. The unsteady aerodynamics are given by the unsteady vortex lattice method, which accounts for wing surfaces subject to large deformations. The implementation of the aerodynamics follows Katz and Plotkin ${ }^{24}$ and the mapping between the aerodynamic and structural grid has been presented by Murua et al. ${ }^{12}$ Only a summary is given here.

\section{III.A. Direct Simulation of Nonlinear Flexible-Aircraft Dynamics}

Time-domain simulation of very flexible aircraft dynamics is obtained via a loosely-coupled implementation of Eq. (3) with the unsteady aerodynamic loads in the external forces, $Q_{\text {ext }}$, given by the UVLM. In the UVLM, vortex-ring quadrilateral elements are used to discretize both lifting surfaces and wakes. Each surface (bound) vortex-ring has an associated circulation strength, $\Gamma_{k}$, and a collocation point, at which the impermeability boundary condition is satisfied. In its general form, the UVLM is a geometrically nonlinear method in which the shape of a force-free wake is obtained as part of the solution procedure. The wake is therefore formed, shed, convected, and allowed to roll up according to the local flow velocity. The vorticity distribution of the vortex elements is determined by applying the non-penetration boundary condition at the discrete time step $n+1$, such that

$$
A_{b} \Gamma_{b}^{n+1}+A_{w} \Gamma_{w}^{n+1}=w^{n+1}
$$

where $\Gamma_{b}$ and $\Gamma_{w}$ are bound (surface) and wake circulation strengths, respectively, $A_{b}$ and $A_{w}$ are the wing-wing and wing-wake aerodynamic influence coefficient matrices. Elements of these matrices are obtained by projecting the velocity (computed using the Biot-Savart law) over the vortex-ring normal vector. The vector of normal components of the non-vortical velocities at the collocation points, $w$, can include deployment of control surfaces, gust- 
induced velocities, wing deformations and rigid-body motions. Note that, as the influence coefficients can account for large deflections of the aerodynamic surfaces, the method is geometrically nonlinear.

Once the vorticity distribution is computed at each time step, the aerodynamic pressures can be computed using Bernoulli's equation. The resulting aerodynamic loads are finally converted into forces and moments at the beam nodes through a mapping procedure as outlined in Murua et al., ${ }^{12}$ which in the current implementation uses coincident meshes. No effort was done in the direct time-simulations in this paper to simplify the aerodynamic description for small wing deformations.

\section{III.B. Monolithic Description of Linearized Flexible-Aircraft Dynamics}

Linearization of the UVLM formulation leads to a compact set of discrete-time state-space equations that, coupled with the fully-linearized form of the flexible-body dynamics EoM, Eq. (4), can be used for linear stability analysis of very flexible aircraft with possibly large deformations at trimmed flight. To obtain the state-space form of the UVLM, the governing equations are linearized on a frozen aerodynamic geometry. ${ }^{20,31}$ They are naturally written in a descriptor state-space form as

$$
\begin{aligned}
E_{F} \Delta x_{F}^{n+1}+F_{F} \Delta u_{F}^{n+1} & =A_{F} \Delta x_{F}^{n}+B_{F} \Delta u_{F}^{n}, \\
\Delta y_{F}^{n} & =C_{F} \Delta x_{F}^{n}+D_{F} \Delta u_{F}^{n},
\end{aligned}
$$

where superscripts $n$ and $n+1$ refer to the current and next time steps and subscript $F$ highlights the aerodynamic components. The outputs $y_{F}$ are the aerodynamic loads at panels and the states $x_{F}$ and inputs $u_{F}$ of the linearized aerodynamic system are given by

$$
x_{F}=\left[\begin{array}{lll}
\Gamma_{b}^{T} & \Gamma_{w}^{T} & \Gamma_{b}^{T}
\end{array}\right]^{T} \quad \text { and } \quad u_{F}=\left[\begin{array}{ll}
\chi_{b}^{T} & \dot{\chi}_{b}^{T}
\end{array}\right]^{T}
$$

where the aerodynamic grid, $\chi_{b}$, and the time derivative, $\dot{\chi}_{b}$, can account for deployment of control surfaces, gust-induced velocities, and wing deformations. This linearized formu- 
lation enables rigid-body motions and elastic deformations to be incorporated in a unified monolithic framework. However, as the linear UVLM is written in discrete time, temporal discretization of the structural equations is also required before the fluid/structure coupling. A standard Newmark- $\beta$ discretization is used, which leads to the following equations for the homogeneous problem ${ }^{32}$

$$
E_{\text {sys }} \Delta x^{n+1}=A_{\text {sys }} \Delta x^{n}
$$

where the state vector that completely determines the linear system is

$$
x=\left[x_{F}^{T}\left|x_{S}^{T}\right| x_{R}^{T}\right]^{T}=\left[\Gamma_{b}^{T} \Gamma_{w}^{T} \dot{\Gamma}_{b}^{T}\left|\eta^{T} \dot{\eta}^{T}\right| \beta^{T} \Theta^{T}\right]^{T}
$$

with the Euler angles, $\Theta$, used to define the orientation of the body frame, $A$. As before, subscripts $S$ and $R$ refer to the structural and rigid-body states. From Eq. (15) one obtains a discrete-time generalized eigenvalue problem to determine the dynamic stability of the vehicle which includes aeroelastic and flight dynamic modes.

\section{Numerical Studies}

Previous work ${ }^{12,16,23}$ has included extensive verification studies of SHARP for static and dynamic solutions of flexible-body configurations, with and without aerodynamics. The linear-flexible/nonlinear-rigid dynamics formulation, outlined in Section II.B, was already exercised in Hesse and Palacios ${ }^{23}$ on configurations with prescribed loads. Those results served to evaluate the limits of applicability of the mean-axes approximation for a multibeam problem in spiral motion.

In this work, we firstly return to the spiral dynamics problem to gain a deeper understanding of the effect of neglecting gyroscopic forces in the mean-axes approximation. Then, a representative flexible HALE aircraft is introduced to exercise the linear-flexible/nonlinearrigid dynamics formulation coupled with the unsteady aerodynamics solver. The $L^{2}$ relative

error norm, defined as $\varepsilon=\left\|X_{\text {com }}-X_{\text {ref }}\right\| /\left\|X_{\text {ref }}\right\|$, will be used throughout this section to 
compare computed results, $X_{\text {com }}$, with a reference solution, $X_{\text {ref }}$.

\section{IV.A. Assessment of the Mean-Axes Approximation on a Flexible Multi-Beam Configuration}

A flexible multi-beam configuration ${ }^{23}$ (FMB) in vacuum will be used to investigate the effect of the gyroscopic forces on the mean-axes approximation. The geometry, material properties, and load history of the FMB problem are defined in Figure 2, where $x-z$ is a plane of symmetry for the model. A set of follower loads is applied at the origin of the bodyfixed frame, $A$, such that the rigid structure follows a circular trajectory with radius $R_{S}$. The parameter $p_{R}$ is introduced in this study to vary the radius of the trajectory which is prescribed by the tangential and centripetal forces, $F_{T}$ and $F_{C}$, respectively, and the moment $M_{C}$. The total mass of the frame is $m$ and $J_{z}$ is the moment of inertia around the $z$ axis at the reference configuration. The body-fixed frame, $A$, is rigidly-linked to the FMB at point $P_{G}$ and the $\mathrm{CM}$ of the undeformed geometry initially coincides with the origin of frame A to simplify comparison with the mean-axes solution. At last, a dead load is applied at $P_{G}$ to prescribe the spiral motion. Note that no gravity forces are acting on the structure in this problem. A mesh size of 60 2-noded elements and a time step of $\Delta t=0.1 \mathrm{~s}$ was found to be sufficient to capture the predominantly rigid-body dynamics for 800 time steps. The constant-acceleration Newmark scheme with algorithmic damping of 0.01 was used to time-march the solution.

The stiffness properties of the structure, shown in Figure 2, result in maximum tip displacements below $1 \%$ relative to the arm length $L$. It was shown before ${ }^{23}$ that for $p_{R}=1$ the relative error (of about 12\%) in tip deformations using the mean-axes approximation is independent of the stiffness even for sufficiently stiff wings. To complete the analysis, this study will investigate the effect of the vehicle kinematics (characterized by radius parameter $\left.p_{R}\right)$ on the error given by the mean-axes approximation.

The trajectory of the centroid is shown in Figure 3 for four different load cases. For this very stiff problem the prescribed forces result in zero angular velocity components around 


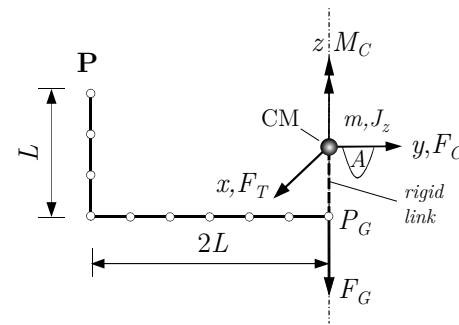

Geometry and material properties:

$L=5 \mathrm{~m}$

$E A=1.75 \times 10^{10} \mathrm{~N}, G A_{s}=5.61 \times 10^{9} \mathrm{~N}$

$G J=3.85 \times 10^{6} \mathrm{Nm}^{2}, E I_{2}=3.65 \times 10^{6} \mathrm{Nm}^{2}, E I_{3}=1.46 \times 10^{7} \mathrm{Nm}^{2}$

$\rho A=13.5 \mathrm{~m}^{2}, J=\operatorname{diag}(1.41,0.28,1.13) \times 10^{2} \mathrm{~m}^{4}$
Follower loads:

$F_{T}(\mathrm{t})=5 \mathrm{~N} / \mathrm{s} \cdot t$

$M_{C}(t)=J_{z} F_{T}(t) /\left(m R_{S}\right)$

$F_{C}(t)=m v_{A y}(t)^{2} / R_{S}$

Dead loads:

$F_{G}(t)=2 \mathrm{~N} / \mathrm{s} \cdot t$

Spiral radius:

$R_{S}=16 L \cdot p_{R}$

Figure 2: Definition of the FMB problem. Cross-sectional properties are given in the local material frame with local $x$ axis acting along the beam reference line and local $y$ axis is normal to $y-z$ plane of body-fixed frame $A$.

$x$ and $y$ axes and the maximum $\omega_{G z}$ component at $t=80 \mathrm{~s}$ is inversely proportional to $p_{R}$. The translational velocity components $v_{G x}$ and $v_{G y}$ are oscillatory due to the circular motion but the norm of $v_{G}$ remains independent of the prescribed spiral radius. This shows that only the inverse proportionality of $p_{R}$ with the angular velocity $\omega_{G z}$ dictates the effect of gyroscopic forces on the tip deflections.

The centrifugal forces result in predominantly antisymmetric in-plane bending of the frame, whereas the resulting normalized tip displacements, shown in Figure 4 for $p_{R}=1$ and 5, remain below $1 \%$ relative to the arm length $L$ defined in Figure 2. Comparison between the nonlinear, Eq. (3), linearized, Eq. (4), and mean-axes solutions, Eq. (10), in Figure 4 demonstrates that the elastic response is linear for this very stiff problem. However, it is clear that application of the mean-axes approximation results in smaller deflections, which converge to the nonlinear solution for larger radii and hence smaller angular velocities.

This behavior is demonstrated in detail in Figure 5 which shows the correlation between the maximum relative error norm of the right tip deflection and its components with the radius parameter $p_{R}$. The error in in-plane bending $\left(R_{A y}\right.$ and $\left.R_{A z}\right)$ contributes the most to the overall relative error norm. It is evident that there is a direct correlation between the angular velocity $\omega_{G z}$, which is inversely proportional to $p_{R}$, and the relative error of the 


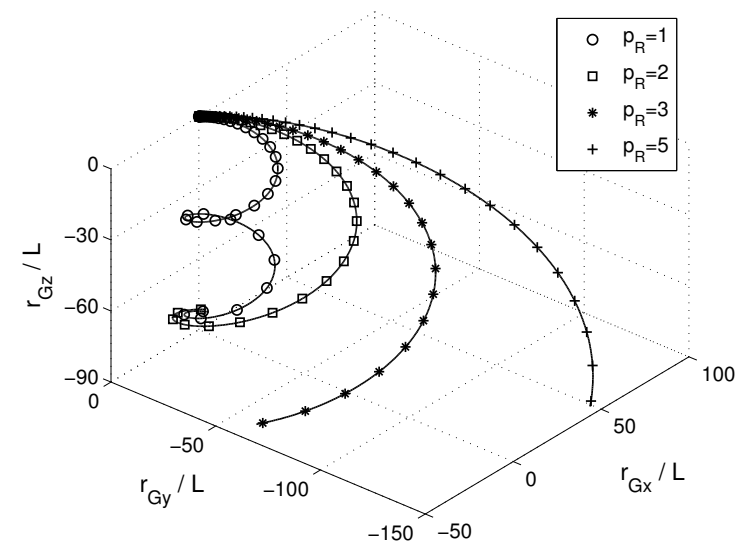

Figure 3: Trajectory of the origin of the body-fixed frame for the FMB problem with different radii. Displacement in the $z$-direction is the same for all cases and markers are plotted every 20 time steps.

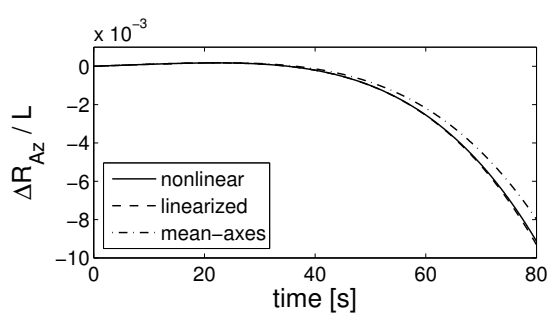

(a) $p_{R}=1$

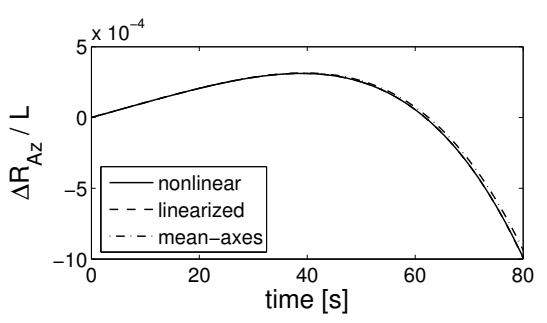

(b) $p_{R}=5$

Figure 4: Vertical displacement of point $\mathrm{P}$, projected in the $A$ frame, for the FMB problem with different radii.

mean-axes approximation to predict the elastic response. The contribution of the gyroscopic forces to damping and stiffness matrices are neglected under the mean-axes approximation in order to decouple the modal system equations. The error of this approximation is dominated by the angular velocity $\omega_{G z}$, as the norm of the translational velocity, $v_{G}$, is constant for all radii. This shows that the gyroscopic forces can have a noticeable impact on the structural dynamics

\section{IV.B. Flight Dynamics of a Flexible Aircraft}

A similar study also including aerodynamic effects will be carried out on a full aircraft configuration. The representative HALE aircraft, shown in Figure 6, consists of large-aspectratio flexible wings, a rigid fuselage and a rigid empennage. It is similar to those of Patil 


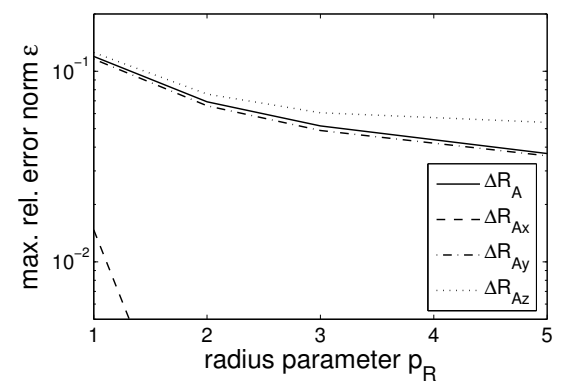

Figure 5: Maximum error norm of displacements at point $\mathrm{P}$ for the FMB problem, comparing mean-axes approximation to the nonlinear solution for different radii.

et al. ${ }^{10}$ and Murua et al., ${ }^{20}$ but it includes dihedral members in the main wing for lateral stability and the point mass of $50 \mathrm{~kg}$ (referred to as payload, even though it is just a first approximation of the non-structural mass) was moved to the center of the main wing to guarantee static pitch stability. The dihedral members are rigidly-linked to the main wing at both ends at an angle of $20 \mathrm{deg}$. The length fraction of each dihedral member over the semi-span of the main wing is defined as $\lambda$, which will be used in this work as a parameter to study the impact of wing dihedral on the vehicle dynamics. The horizontal stabilizer and the vertical fin are modeled as lifting surfaces without camber or pretwist, and the former includes an elevator modeled as a quarter-chord-length control surface. The empennage is raised by $1.25 \mathrm{~m}$ with respect to the main wing to avoid wake-tail collisions ${ }^{32}$ and it is rigidly linked to the main wing by a non-lifting fuselage.

This basic aircraft configuration also includes two massless propellers, which are modeled as point forces rigidly linked to the main wing. The mass per unit length of the fuselage is the same as that of the horizontal and vertical tail planes, and thus the total mass of this aircraft, including payload and structural mass, is $75.4 \mathrm{~kg}$. The flight conditions are $V_{\infty}=30 \mathrm{~m} / \mathrm{s}$ at an altitude of $20 \mathrm{~km}$ (air density $0.0889 \mathrm{~kg} / \mathrm{m}^{3}$ ). The relevant properties of the members are listed in Table 2. The stiffness parameter $\sigma$ will be used in the following studies to vary the stiffness properties of the main wing (decreasing $\sigma$ results in a more flexible wing). 

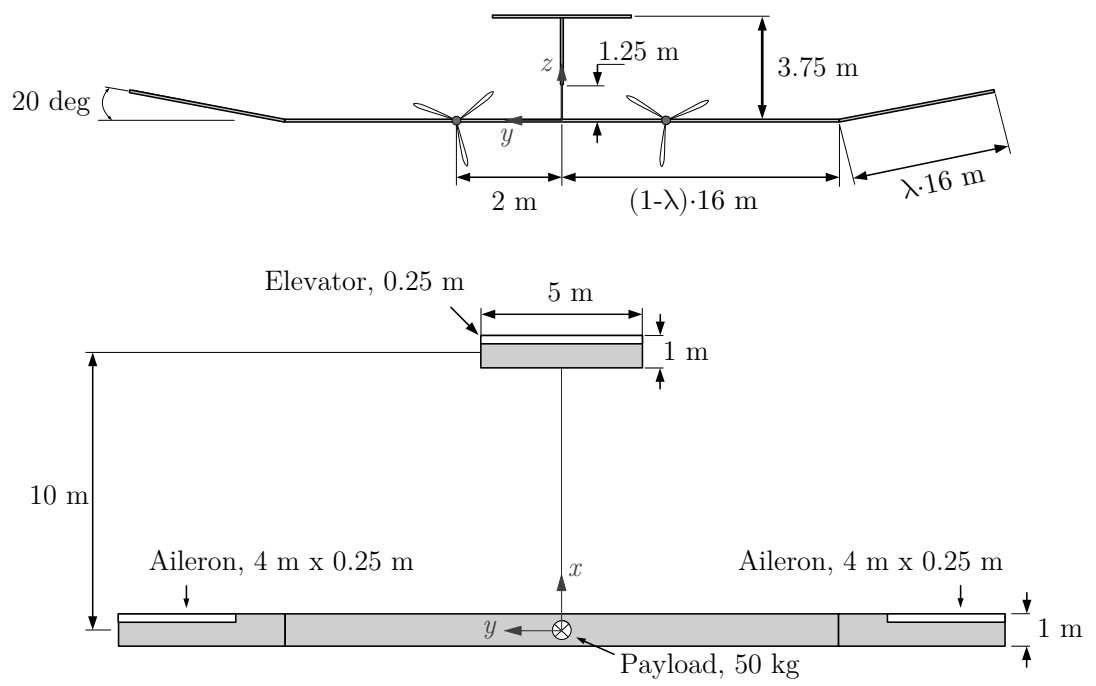

Figure 6: Undeformed HALE aircraft geometry with front and top view (not to scale).

Table 2: HALE aircraft properties.

\begin{tabular}{lll}
\hline \hline & Main wing & Tail plane \\
\hline Chord & $1 \mathrm{~m}$ & $0.5 \mathrm{~m}$ \\
Semi-span for $\lambda=0, B$ & $16 \mathrm{~m}$ & $2.5 \mathrm{~m}$ \\
Elastic axis & $50 \%$ chord & $50 \%$ chord \\
Center of gravity & $50 \%$ chord & $50 \% \mathrm{chord}$ \\
Mass per unit length & $0.75 \mathrm{~kg} / \mathrm{m}$ & $0.08 \mathrm{~kg} / \mathrm{m}$ \\
Moment of inertia & $0.1 \mathrm{~kg} \cdot \mathrm{m}$ & $0.01 \mathrm{~kg} \cdot \mathrm{m}$ \\
Torsional stiffness & $1 \sigma \times 10^{4} \mathrm{~N} \cdot \mathrm{m}^{2}$ & $\infty$ \\
Bending stiffness & $2 \sigma \times 10^{4} \mathrm{~N} \cdot \mathrm{m}^{2}$ & $\infty$ \\
In-plane bending stiffness & $4 \sigma \times 10^{6} \mathrm{~N} \cdot \mathrm{m}^{2}$ & $\infty$ \\
\hline \hline
\end{tabular}

\section{IV.B.1. Trim for steady flight}

As a first step, the vehicle is trimmed for steady level flight through three inputs, namely angle of attack, elevator deflection, $\delta_{e}^{\text {trim }}$, and thrust per propeller. The trimming method is a tightly-coupled process that is described in detail in Murua et al. ${ }^{20}$ The trim characteristics of the HALE aircraft are presented in Figure 7 for dihedral member ratio, $0 \leq \lambda \leq 1 / 2$, and stiffness parameter of the main wing, $1.1 \leq \sigma \leq 1000$. The tip deflection of the main wing, non-dimensionalized with the overall semi-span of the main wing, $B$, is also included in Figure 7. Parameter $\sigma=1$ corresponds to the original stiffness properties from Patil et 

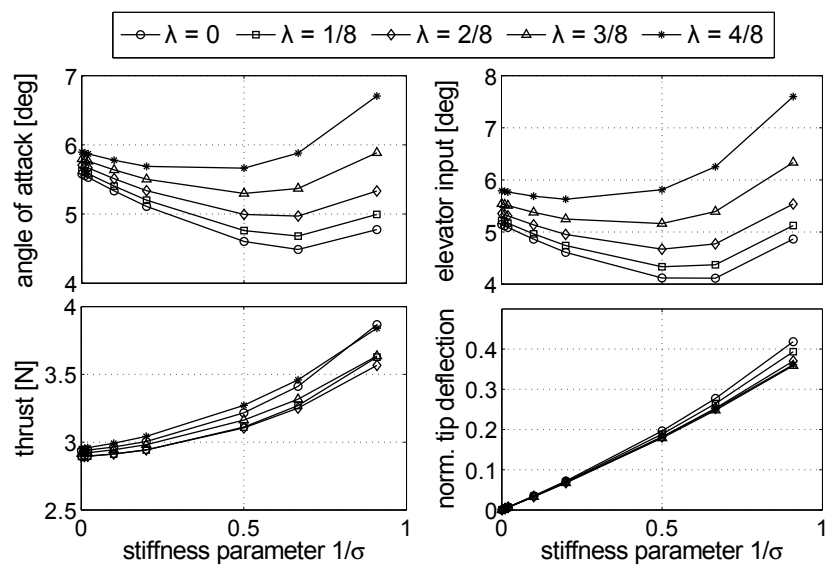

Figure 7: Trim angle of attack, elevator deflection, thrust per propeller, and vertical tip deflection of the main wing, $\Delta R_{A z} / B$, as a function of wing flexibility, $\sigma$, and dihedral member ratio, $\lambda$.

al. ${ }^{10}$ but it gives tip deflections over $40 \%$ at current flight conditions which were deemed unrealistic. It can be seen that the angle of attack initially drops with increasing flexibility due to favorable twisting of the wing. However, this is opposed by wing bending for very flexible configurations $(\sigma \geq 2)$ resulting in loss of vertical forces because of the additional wing dihedral and illustrates the importance of geometrically nonlinear effects on the aircraft trim characteristics. Similarly, the increasing span ratio of the dihedral members, $\lambda$, is also compensated by a larger angle of attack and elevator deflection. The wing tip deflection decreases for increasing values of $\lambda$ due to the decrease in bending moment from the dihedral members, as the lift vector is pointing inwards. Figure 8 shows the trim deformations of the HALE configuration for a range of stiffness values $\sigma$ and for $\lambda=1 / 2$, and demonstrates the large effective wing dihedral for the very flexible aircraft.

\section{IV.B.2. Asymptotic Stability Analysis}

The dynamic stability of this HALE configuration is studied next to investigate the effect of dihedral member ratio $(\lambda)$ and flexibility $(\sigma)$ on the vehicle flight dynamics. The stability characteristics will be subsequently compared to the open-loop response of the aircraft. For each trim solution, we can directly solve the generalized eigenvalue problem posed by the linear aeroelastic system coupled with the flight dynamics of the flexible aircraft, derived 


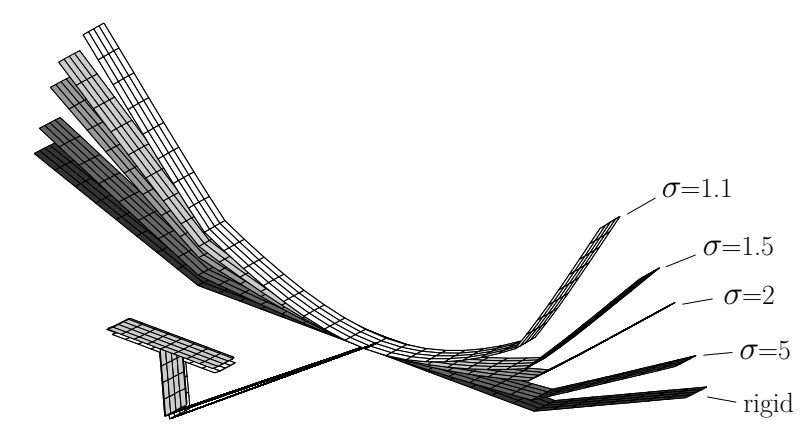

Figure 8: Trim deformation of HALE aircraft with $\lambda=1 / 2$ and different stiffness parameters $\sigma$. (Figure shows actual deformations.)

from Eq. (15). The aerodynamic mesh used to solve this problem is shown in Figure 8. The wake is cut off after $20 \mathrm{~m}$ with a time step of $0.01 \mathrm{~s}$. The beam elements coincide with the aerodynamic panels in the spanwise direction. The resulting total system size is 1581 states which includes 924 aerodynamic states, 648 structural states, and the 9 rigid-body states.

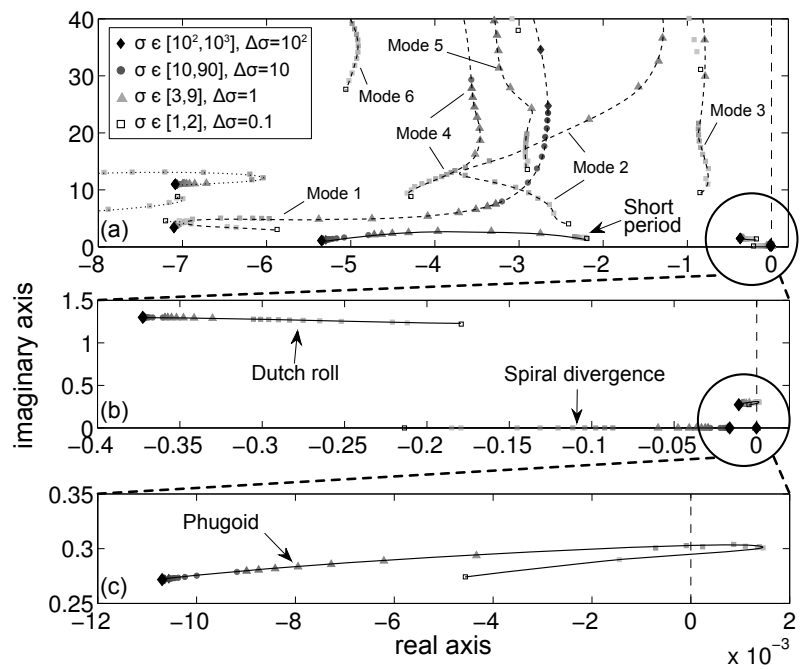

Figure 9: Root loci of the eigenvalue analysis for $\lambda=1 / 4$ with stiffness of the main wing as parameter: (a) dominant flight dynamic (solid) and aeroelastic (dashed) modes, and (b-c) zooms showing flight dynamic modes close to the origin. Aerodynamic modes highlighted with dotted curves.

Figure 9 presents the root loci as a function of the wing stiffness, $\sigma$, for a dihedral ratio of $\lambda=1 / 4$. Note that the discrete-time eigenvalue analysis derived from Eq. (15) yields as many roots as the number of states in the problem, but only the dominant modes are 
presented here. The first few coupled modes of the flexible vehicle have been identified and are designated by the names of their rigid-aircraft counterparts, e.g., phugoid, short period, etc., although they also include vehicle deformations. The aeroelastic modes are labeled with numbers where Modes 1, 2, and 5 have been identified as symmetric bending/torsion modes and Modes 3, 4, and 6 correspond to the anti-symmetric modes. Aerodynamic modes can also be captured with this stability analysis but they are highly damped and mostly fall beyond the boundaries of Figure 9(a). The stiffness values have been unevenly spaced, as defined in Figure 9(a), where the two extreme cases are representative of a very flexible $(\sigma=1)$ and a stiff $(\sigma=1000)$ wing. Note that the discrete-time eigenvalues have been converted to continuous time for an easier interpretation.

Figure 9(a) demonstrates that for very flexible aircraft the frequency separation between flight dynamic and aeroelastic modes can disappear. As the stiffness of the main wing is increased, the higher-frequency aeroelastic modes become less stable while the low-frequency flight dynamic modes show the opposite trend. The non-oscillatory spiral divergence on the other hand, becomes less stable with increasing stiffness. This can be observed in detail in Figure 9(b) which highlights two flight-dynamics lateral modes: Dutch roll and spiral divergence. As flexibility increases, the less stable Dutch roll illustrates the detrimental impact flexibility might have on the handling qualities of the vehicle. In contrast, the spiral mode stability margin substantially increases with flexibility due to the larger static deformations of the main wing at trim conditions.

Figure $9(\mathrm{c})$ zooms further in the root loci in order to visualize the phugoid mode. For a rigid aircraft it is a lightly damped mode. As flexibility is increased, this modes demonstrates the complex interaction between the elastic and rigid-body DoF: as the aircraft pitches up and climbs first, the wings undergo a downward flapping-like motion. As the vehicle pitches down and descends, the wing bends upwards, reaching the maximum tip deflections before the cycle is finished. Whereas rigid and stiff aircraft present stable phugoid modes, damping drops with increasing wing flexibility resulting in an instability for $1.2 \leq \sigma \leq 1.7$. However, for highly flexible cases $(\sigma<1.2)$ this trend is reversed which results in stable configurations. 
To investigate this reversal in stability behavior, we have included the extremely flexible case of $\sigma=1$ in Figure 9 and will examine the effect of the outboard dihedral in the following.

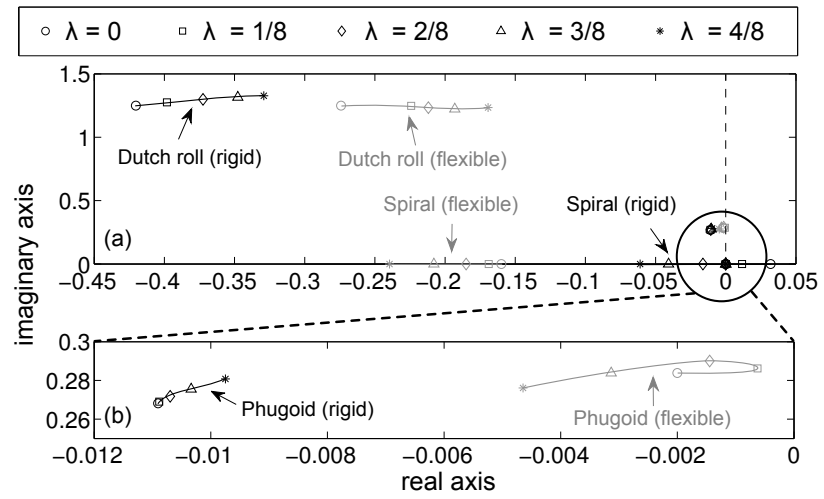

Figure 10: Root loci of the eigenvalue analysis for $\sigma=1.1$ ("flexible") and $\sigma=1000$ ("rigid") with the dihedral ratio $\lambda$ as parameter: (a) dominant roots, and (b) zoom near to the origin.

Figure 10 shows the root loci for a very stiff $(\sigma=1000)$ and a very flexible $(\sigma=1.1)$ aircraft for varying dihedral ratio, $\lambda$. Here, we only focus on the dominant flight dynamic modes: Dutch roll, spiral divergence, and phugoid. Figure 10(a) shows that an increase of the dihedral member ratio leads to a less stable Dutch roll, while it increases the resistance against spiral divergence. This coincides with the trends observed in Figure 9(a) with increased flexibility. That is, static deformations and dihedral ratio have the same effect on the lateral modes. As the dihedral ratio is varied, these trends are consistent for both the rigid and flexible vehicles. As expected, wings with very small or no dihedral lead to unstable spiral modes.

Figure 10(b) focuses on the region closer to the origin in order to expose the characteristics of the phugoid mode. Interestingly, the trends are opposite for the rigid and flexible vehicles: the increase of dihedral ratio leads to a more stable phugoid in the flexible aircraft (except for $\lambda=0$ ), but the damping of the rigid phugoid decreases with $\lambda$. From the trends of the phugoid mode in Figures 9 and 10, it is clear that static and dynamic aeroelastic effects have opposing contributions, such that an increase of effective wing dihedral due to wing bending (static effect) decreases phugoid stability, as deduced from Figure 10, which explains the 
trend for $1.2 \leq \sigma \leq 1.7$ in Figure 9. For more flexible configurations the dynamic properties result in increased damping which dominate over the static effects and tend to stabilize the aircraft.

\section{IV.B.3. Open-Loop Response of HALE Aircraft with Large Rigid-Body Motions}

Finally, we can investigate the effect of consistent linearization of the structural DoF on the dynamic response of the vehicle defined in Figure 6 and Table 2. The aircraft is subject to various excitations in the form of commanded inputs on ailerons and elevator and the open-loop response is computed using the linearized and mean-axes solutions, as well as the full nonlinear solver. To isolate the effect of linearization of the structural DoF only, the aerodynamics were computed using the geometrically nonlinear time-marching description of the UVLM for all results presented here. The stiffness parameter $\sigma$ is also used in this study to explore the effect of flexibility on the nonlinear flight dynamic response.

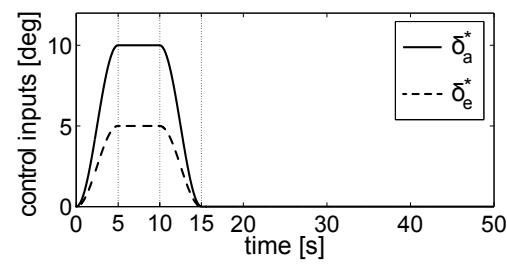

Figure 11: Control inputs for ailerons $\left(\delta_{a}^{*}\right)$ and elevator $\left(\delta_{e}^{*}\right)$. Transients are sine functions.

We will limit ourselves to the configuration with dihedral member ratio $\lambda=1 / 4$, which was shown to be stable for the whole range of stiffness values with lightly-damped spiral and phugoid modes (see Figures 9-10). For the dynamic analysis, the control surface inputs of ailerons and elevator (denoted by subscripts $a$ and $e$, respectively) are prescribed such that $\delta_{a}= \pm \delta_{a}^{*}$ and $\delta_{e}=\delta_{e}^{t r i m}+\delta_{e}^{*}$ with the time histories of $\delta_{a, e}^{*}$ shown in Figure 11. The elevator inputs are prescribed around the trim inputs, $\delta_{e}^{\text {trim }}$, while the ailerons, as defined in Figure 6 , are not used for trimming the aircraft but are deployed anti-symmetrically with $\pm \delta_{a}^{*}$. The open-loop response of the aircraft is computed for $50 \mathrm{~s}$ using a time step of $0.025 \mathrm{~s}$.

Figure 12(a) shows the angular velocity components of the rigid vehicle expressed in the body-fixed frame $A$. The time history of the angular velocity norm, $\left|\omega_{A}\right|$, indicates that the 


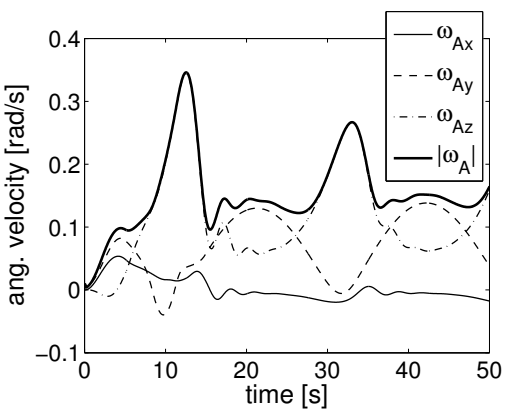

(a)

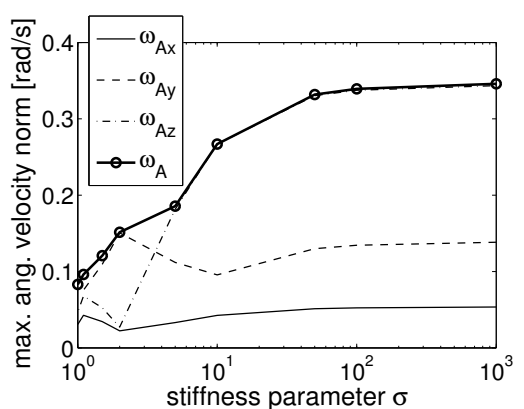

(b)

Figure 12: Rigid-body angular velocity components of the HALE aircraft: (a) time history of the rigid vehicle and (b) maximum norm of $\omega_{A}$ and its individual components for varying stiffness computed using the geometrically nonlinear model.

aircraft response remains lightly damped even long after the command $(t \geq 20 \mathrm{~s})$. This finding is in agreement with the above stability analysis in Figure 9, which suggests that the rigid aircraft is only marginally stable. It was also shown, however, that the spiral mode becomes more stable with increasing flexibility due to wing dihedral. To demonstrate this behavior Figure 12(b) compares the maximum norm of the angular velocity $\omega_{A}$ and its components for different values of the flexibility parameter, $\sigma$. The yaw rate, $\omega_{A z}$, is damped much faster for the flexible platforms $(\sigma<5)$ as the response is dominated by the less stable phugoid mode in this flexibility range.

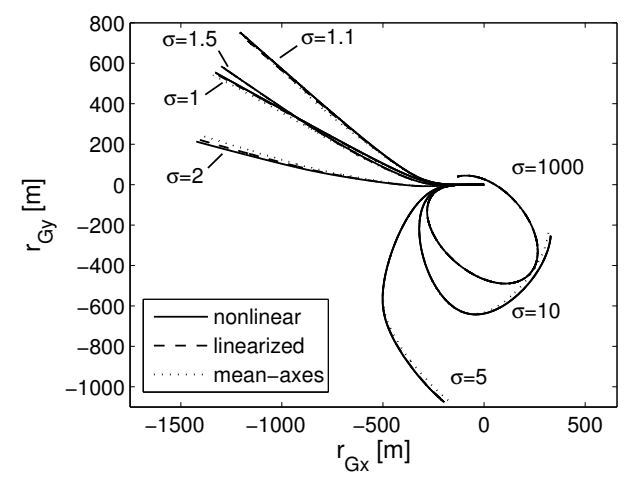

(a) $x-y$ plane using different models

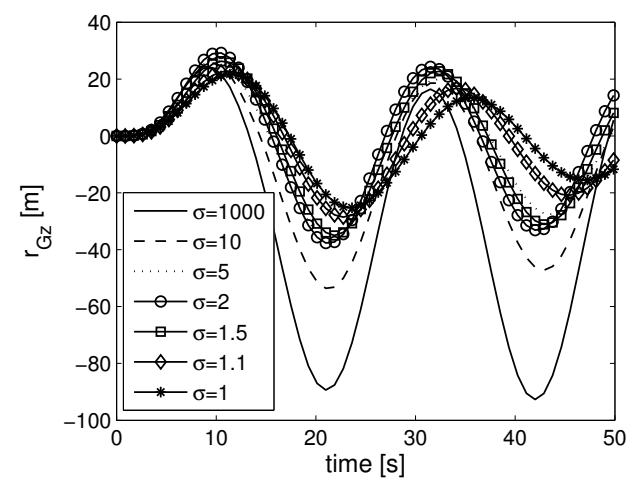

(b) Time history of altitude, $z$, using the geometrically nonlinear model

Figure 13: Trajectory of the HALE aircraft for $\lambda=1 / 4$ with stiffness of the main wing as parameter.

In addition to the stability characteristics, flexibility impacts the controllability of the 
aircraft. Figure 13(a) shows its trajectory in the $x-y$ plane (top view) for different stiffness values $\sigma$ and demonstrates control reversal with increasing flexibility of the main wing. For the flexible configuration with $\sigma=2$, wing twisting results in minimal control authority of the ailerons. Controllability can be further explored on the linear system by evaluating the controllability grammians, ${ }^{33}$ but this will not be investigated here. Figure 13(b) shows the effect of flexibility on the altitude response of the HALE aircraft. The frequency of the resulting oscillatory motion matches closely the phugoid mode presented for this configuration in Figure 9. The reduction in frequency with increasing flexibility is also captured in this response.

To compare the effect of linearization of the elastic DoF, Figure 13(a) also presents the dynamic behavior obtained using the linear-flexible/nonlinear-rigid formulation, Eq. (4), which suggests that geometrically nonlinear deformations have a negligible effect on the aircraft motion in the $x-y$ plane. The effect of geometric nonlinearity is very different in the altitude response of the aircraft. This is demonstrated in Figure 14, which shows the maximum error of the linearized formulation to compute the vertical position for varying flexibility of the main wing. The maximum norm of the HALE aircraft altitude was used to normalize the error for each $\sigma$. The linearized solution accurately captures the rigid-body response for mildly-flexible vehicles ( $\sigma \geq 5$ corresponding to $7 \%$ wing tip deflections), but the geometrically nonlinear deformations dominate for more flexible configurations.

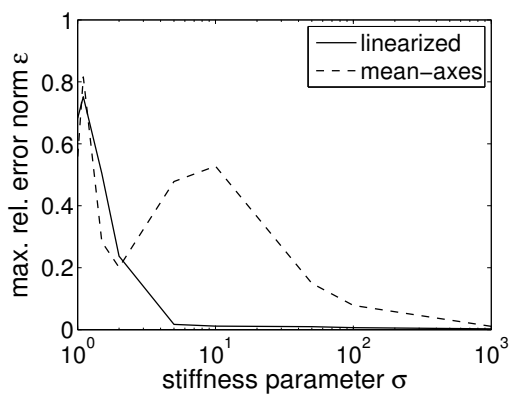

Figure 14: Maximum relative error norm of aircraft altitude using linearized and mean-axes approximations compared to the nonlinear solution for $\lambda=1 / 4$ and varying stiffness $\sigma$.

Next, the open-loop response of the HALE aircraft was computed using the mean-axes 
approximation, Eq. (10), but the aerodynamics in our implementation are still computed on the actual instantaneous geometry. For small deformations this converges to the classical mean-axes approximation as it is commonly found in flight dynamic simulations. ${ }^{4}$ The mean-axes solution to the rigid-body response of the HALE aircraft is included in Figures 13-14 and compared with the fully-coupled, nonlinear reference solution. The error norm in Figure 14 gives an insight into the effect of neglecting the gyroscopic forces due to elastic deformations. One would expect the error to increase with flexibility, as is the case for $\sigma<2$, but a local maximum occurs at $\sigma=10$ which represents a rather stiff configuration with $5 \%$ maximum wing deformations. We saw from Figure 12(b) that the angular velocity of the aircraft, and hence the contribution of the gyroscopic forces, reduces with increasing flexibility. This competition between the opposing effects of gyroscopic forces and flexibility results in the peak at around $\sigma=10$.

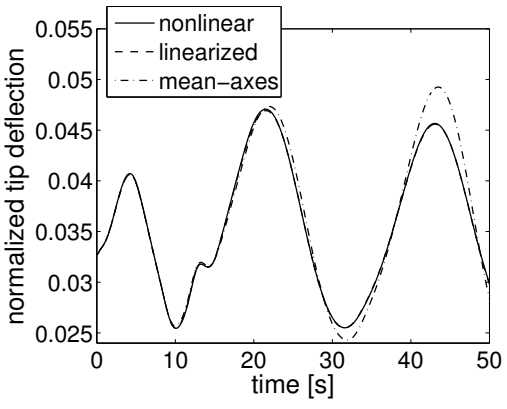

(a) $\sigma=10$

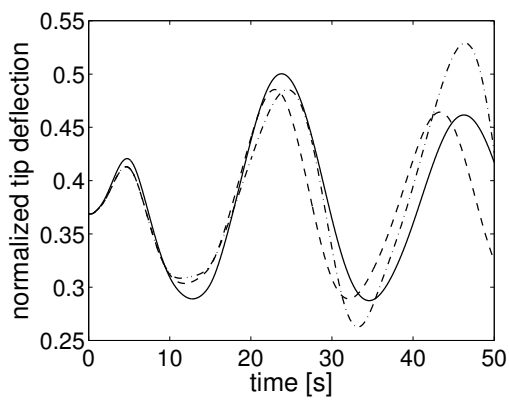

(b) $\sigma=1.1$

Figure 15: Vertical tip deflection of the right main wing normalized with the semi-span of $B=16$ s for dihedral ratio of $\lambda=1 / 4$.

The right wing tip deflection is presented in Figure 15 for a stiff $(\sigma=10)$ and a very flexible $(\sigma=1.1)$ aircraft. Whereas the flexible case exhibits static equilibrium deformations of $37 \%$ of the semi-span, the maximum amplitude in the transient around this equilibrium is below $10 \%$ of the semi-span. As a result, the linearized formulation is able to capture the amplitudes of the large deformations well even for the very flexible problem, which is presented in Figure 15(b). However, as the mass matrix is assumed constant in the linearized approach, the change in geometry due to the deformations and the resulting change in sta- 
bility behavior of the aircraft cannot be captured which results in a frequency shift. Figure 16 shows the maximum error norm of the vertical wing tip displacement which were normalized with the maximum wing deformations for each $\sigma$. The linearized approach predicts the displacements well even for very flexible aircraft with $\sigma=2$ and wing deformations of up to $26 \%$ of the semi-span. For more flexible configurations the resulting phase shift due to geometric nonlinearity effects dominate.

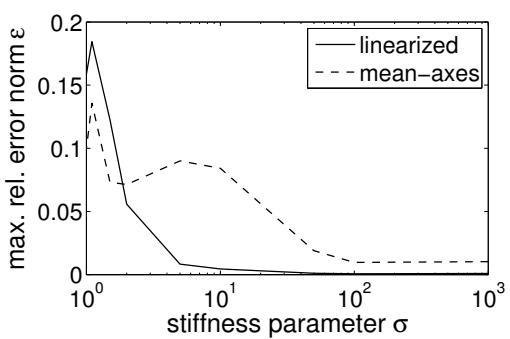

Figure 16: Maximum relative error norm of vertical wing tip deflections for $\lambda=1 / 4$ and varying $\sigma$.

At last, it is shown that the mean-axes approximation tends to overpredict the elastic deformations in the open-loop dynamics. Figure 15(a) indicates that even for relatively stiff problems $(\sigma=10)$, corresponding to maximum deflections below $5 \%$ of the semi-span, the mean-axes approximations results in errors around $9 \%$ compared to the nonlinear reference solution. Similar to the FMB problem, Figure 16 shows that even for near-rigid vehicles $(\sigma \geq 100)$ the relative error remains constant around $1 \%$ for the commanded maneuver. Whereas it was shown in Figure 14 that the mean-axes approximation can give a good estimate of the open-loop flight dynamic response for very stiff structures, it results in unreliable predictions of the elastic deformations even for very stiff aircraft. This makes it less suitable for the prediction of dynamic maneuver and gust loads.

\section{Conclusions}

This paper has presented a coupled aeroelastic and flight dynamics framework for flexible aircraft with a consistent linearization of the structural dynamics around a nonlinear 
static trim equilibrium. Starting from a displacement-based geometrically nonlinear composite beam model, coupled with an unsteady vortex lattice method, linearization in only the structural degrees of freedom has resulted in flexible-body dynamics equations written in matrix form, with coefficients that vary only with the vehicle rigid-body velocities. Approximating the finite-element discretization in terms of a few vibration modes of the unconstrained structure has been shown to result in closed-form expressions for the equations of motion with constant coefficients that capture the gyroscopic couplings between the rigidbody and the structural dynamics. The size of the resulting system is thus comparable to that in mean-axes approximations, but it includes the gyroscopic couplings and vehicle dynamics which are written around a nonlinear aeroelastic equilibrium - including a deformed aerodynamic lattice. Moreover, it is also not necessary to pre-compute frequency-domain aerodynamics, as it is done in solutions based on the doublet lattice method.

Numerical results on a free-flying flexible multi-beam configuration have shown that the mean-axes approximation results in a constant relative error independent of the extent of deformations. More importantly, this error correlates directly to the angular velocity of the flexible body. Neglecting the gyroscopic couplings may therefore have an important effect in the dynamic maneuver loads. To test that, the proposed linearized framework has also been exercised on a representative HALE configuration subject to commanded lateral control inputs. A linear stability analysis and the open-loop response of the aircraft have confirmed the dramatic effect of flexibility on the vehicle dynamics, and this was shown to be adequately captured even for rather flexible configurations using the linear-flexible/nonlinearrigid model. It has finally been shown that the mean-axes approximation fails to predict appropriately the elastic deformations in this open-loop response even for stiff aircraft.

In summary, the linearized flexible-aircraft formulation provides a powerful tool to accurately describe the flight dynamics of current and next-generation high-aspect-ratio aircraft. The proposed model captures all coupling terms due to gyroscopic forces, which implies that the resulting linear elastic deformations can be captured accurately. This may be critical for the prediction of maneuver loads on flexible vehicles, and it has only a small computational 
burden due to the modal projection of the structural degrees of freedom.

\section{Acknowledgments}

The work of Henrik Hesse is sponsored by the UK Engineering and Physical Sciences Research Council (EPSRC). This support is gratefully acknowledged.

\section{References}

${ }^{1}$ Milne, R. D., "Dynamics of the Deformable Plane," Tech. Rep. 3345, Her Majesty's Stationary Office, London, UK, Sept. 1962.

${ }^{2}$ Agrawal, O. P. and Shabana, A. A., "Application of deformable-body mean axis to flexible multibody system dynamics," Computer Methods in Applied Mechanics and Engineering, Vol. 56, No. 2, 1986, pp. 217245 .

${ }^{3}$ Friedmann, P. P., McNamara, J. J., Thuruthimattam, B. J., and Nydick, I., "Aeroelastic analysis of hypersonic vehicles," Journal of Fluids and Structures, Vol. 19, No. 5, 2004, pp. 681-712.

${ }^{4}$ Schmidt, D. K., Modern Flight Dynamics, McGraw-Hill, 2011.

${ }^{5}$ Rodden, W. P. and Love, J. R., "Equations of Motion of a Quasisteady Flight Vehicle Utilizing Restrained Static Aeroelastic Characteristics," Journal of Aircraft, Vol. 22, No. 9, 1985, pp. 802-809.

${ }^{6}$ Meirovitch, L. and Tuzcu, I., "Unified theory for the dynamics and control of maneuvering flexible aircraft," AIAA Journal, Vol. 42, No. 4, 2004, pp. $714-27$.

${ }^{7}$ Meirovitch, L. and Tuzcu, I., "The Lure of the Mean Axes," Journal of Applied Mechanics, Vol. 74, No. 3, 2007, pp. 497-504.

${ }^{8}$ Haghighat, S., Martins, J. R. R. A., and Liu, H. H. T., "Aeroservoelastic Design Optimization of a Flexible Wing," Journal of Aircraft, Vol. 49, No. 2, 2012, pp. 432-443.

${ }^{9}$ Drela, M., "Integrated simulation model for preliminary aerodynamic, structural, and control-law design of aircraft," 40th AIAA/ASME/ASCE/AHS/ASC Structures, Structural Dynamics and Materials Conference, AIAA Paper 1999-1394, St. Louis, Missouri, USA, 1999.

${ }^{10}$ Patil, M. J., Hodges, D. H., and Cesnik, C. E., "Nonlinear aeroelasticity and flight dynamics of highaltitude long-endurance aircraft," Journal of Aircraft, Vol. 38, No. 1, 2001, pp. 88-94.

${ }^{11}$ Wang, Z., Chen, P. C., Liu, D. D., and Mook, D. T., "Nonlinear-Aerodynamics/Nonlinear-Structure 
Interaction Methodology for a High-Altitude Long-Endurance Wing," Journal of Aircraft, Vol. 47, No. 2, 2010, pp. 556-566.

${ }^{12}$ Murua, J., Palacios, R., and Graham, J. M. R., "Assessment of Wake-Tail Interference Effects on the Dynamics of Flexible Aircraft," AIAA Journal, Vol. 50, No. 7, July 2012, pp. 1575-1585.

${ }^{13}$ Shearer, C. M. and Cesnik, C. E., "Nonlinear Flight Dynamics of Very Flexible Aircraft," Journal of Aircraft, Vol. 44, No. 5, 2007, pp. 1528-1545.

${ }^{14} \mathrm{Su}, \mathrm{W}$. and Cesnik, C., "Nonlinear Aeroelasticity of a Very Flexible Blended-Wing-Body Aircraft," Journal of Aircraft, Vol. 47, No. 5, 2010, pp. 1539-1553.

${ }^{15}$ Cesnik, C. E. and Brown, E. L., "Modeling of high aspect ratio active flexible wings for roll control," 43rd AIAA/ASME/ASCE/AHS/ASC Structures, Structural Dynamics and Materials Conference, AIAA 2002-1719, Denver, CO, USA, 2002.

${ }^{16}$ Palacios, R., Murua, J., and Cook, R., "Structural and Aerodynamic Models in the Nonlinear Flight Dynamics of Very Flexible Aircraft," AIAA Journal, Vol. 48, No. 11, 2010, pp. 2648-2559.

${ }^{17}$ Hodges, D. H., "Geometrically exact, intrinsic theory for dynamics of curved and twisted anisotropic beams," AIAA Journal, Vol. 41, No. 6, 2003, pp. 1131-1137.

${ }^{18}$ Sotoudeh, Z. and Hodges, D. H., "Incremental Method for Structural Analysis of Joined-Wing Aircraft," Journal of Aircraft, Vol. 48, No. 5, 2011, pp. 1588-1601.

${ }^{19}$ Palacios, R., "Nonlinear normal modes in an intrinsic theory of anisotropic beams," Journal of Sound and Vibration, Vol. 330, No. 8, 2011, pp. 1772-1792.

${ }^{20}$ Murua, J., Palacios, R., and Graham, J. M. R., "Applications of the unsteady vortex-lattice method in aircraft aeroelasticity and flight dynamics," Progress in Aerospace Sciences, Vol. 55, Nov. 2012 , pp. 46-72.

${ }^{21}$ Ronch, A. D., Badcock, K. J., Wang, Y., Wynn, A., and Palacios, R., "Nonlinear Model Reduction for Flexible Aircraft Control Design," AIAA Atmospheric Flight Mechanics Conference, AIAA Paper 2012-4404, Minneapolis, MI, USA, 2012.

${ }^{22} \mathrm{Su}$, W. and Cesnik, C. E. S., "Strain-Based Analysis for Geometrically Nonlinear Beams: A Modal Approach," 53 $3^{\text {rd }} A I A A / A S M E / A S C E / A H S / A S C$ Structures, Structural Dynamics, and Materials Conference, Honolulu, HI, USA, 2012.

${ }^{23}$ Hesse, H. and Palacios, R., "Consistent structural linearisation in flexible-body dynamics with large rigid-body motion," Computers $\&$ Structures, Vol. 110-111, 2012, pp. 1-14.

${ }^{24}$ Katz, J. and Plotkin, A., Low-Speed Aerodynamics, Cambridge Aerospace Series, Cambridge University Press, New York, NY, USA, 2nd ed., 2001.

${ }^{25}$ Simo, J. C. and Vu-Quoc, L., "On the dynamics in space of rods undergoing large motions - A 
geometrically exact approach," Computer Methods in Applied Mechanics and Engineering, Vol. 66, No. 2, 1988, pp. $125-161$.

${ }^{26}$ Géradin, M. and Cardona, A., Flexible Multibody Dynamics: A Finite Element Approach, John Wiley \& Sons Ltd, Chichester, UK, 2001.

${ }^{27}$ Hodges, D., "A mixed variational formulation based on exact intrinsic equations for dynamics of moving beams," International Journal of Solids and Structures, Vol. 26, No. 11, 1990, pp. 1253-1273.

${ }^{28}$ Stevens, B. L. and Lewis, F. L., Aircraft Control and Simulation, John Wiley \& Sons, Inc., New York, NY, USA, 1992.

${ }^{29}$ Géradin, M. and Rixen, D., Mechanical Vibrations: Theory and Application to Structural Dynamics, John Wiley \& Sons Ltd, Chichester, UK, 2nd ed., 1997.

${ }^{30}$ Waszak, M. R. and Schmidt, D. K., "Flight dynamics of aeroelastic vehicles," Journal of Aircraft, Vol. 25, No. 6, 1988, pp. 563-571.

${ }^{31}$ Hall, K., "Eigenanalysis of Unsteady Flows about Airfoils, Cascades and Wings," AIAA Journal, Vol. 32, No. 12, 1994, pp. 2426-2432.

${ }^{32}$ Murua, J., Palacios, R., and Graham, J. M. R., "Open-Loop Stability and Closed-Loop Gust Alleviation on Flexible Aircraft Including Wake Modeling," 53rd AIAA/ASME/ASCE/AHS/ASC Structures, Structural Dynamics, and Materials Conference, AIAA 2012-1484, Honolulu, HI, USA, 2012.

${ }^{33}$ Moore, B., "Principal component analysis in linear systems: Controllability, observability, and model reduction," IEEE Transactions on Automatic Control, Vol. 26, No. 1, 1981, pp. 17-32. 